\title{
Properties of Piriform Cortex Pyramidal Cell Dendrites: Implications for Olfactory Circuit Design
}

\author{
Brice Bathellier, ${ }^{1}$ Troy W. Margrie, ${ }^{2}$ and Matthew E. Larkum ${ }^{1}$ \\ ${ }^{1}$ Department of Physiology, University of Bern, CH-3012 Bern, Switzerland, and ${ }^{2}$ Department of Neuroscience, Physiology, and Pharmacology, University \\ College London, London WC1E 6BT, United Kingdom
}

\begin{abstract}
Unlike the neocortex, sensory input to the piriform cortex is anatomically segregated in layer 1, making it ideal for studying the dendritic integration of synaptic inputs pivotal for sensory information processing. Here we investigated dendritic integration of olfactory bulb inputs in pyramidal cells using dual patch-clamp recordings along the soma-apical dendritic axis. We found that these dendrites are relatively compact with $50 \%$ maximal somatic current loss for synaptic inputs arriving at distal dendritic regions. Distal dendrites could generate small and fast local spikes, but they had little impact on the soma, indicating that they are only weakly active. In contrast to the neocortex, we found no evidence for dendritic $\mathrm{Ca}^{2+}$ or NMDA spikes though these dendrites actively supported action potential backpropagation with concomitant entry of $\mathrm{Ca}^{2+}$ ions. Based on experiments and simulations we suggest that regardless of dendritic location, olfactory bulb inputs have nearly uniform potency and are distributed diffusely over the distal apical tree (layer Ia), thereby minimizing sublinear summation effects. This indicates that any stimulus feature extraction performed by these cells will occur at the soma and is based on the nearly linear sum of olfactory bulb inputs, rather than on explicitly designed clusters of functionally related synapses in the dendritic tree.
\end{abstract}

\section{Introduction}

Like the hippocampus, the piriform cortex is a three-layered structure in which the principal excitatory neurons are pyramidal cells. Their cell bodies are found in layer II (superficial pyramidal cells) and in layer III (deep pyramidal cells), while a third population of smaller excitatory semilunar cells is found in the superficial portion of layer II (Fig. 1A) (Suzuki and Bekkers, 2006). One attractive feature of the piriform cortical slice preparation is that functionally distinct inputs from the olfactory bulb via the lateral olfactory tract (LOT) synapse almost exclusively on the distal apical dendrites in layer Ia (Arbib et al., 1998). The information arriving through theses distal synapses provides the vast majority of olfactory signals to the cortex that is presumably used for sensory tasks such as odor discrimination and recognition.

The encoding of odors in the mammalian olfactory system essentially involves different combinations of receptor activations, which result in complex spatiotemporal firing patterns of many output cells in the olfactory bulb (Margrie and Schaefer, 2003; Lin et al., 2005; Mori et al., 2006; Bathellier et al., 2008). These patterns need to be precisely recognized by the downstream structures to allow efficient olfactory perception. Theoretical models of odor recognition generally suppose that appropriate connectivity allows specific pyramidal cells in the piriform cortex to fire in response to the coactivation of specific combinations of olfac-

\footnotetext{
Received March 5, 2009; revised Aug. 5, 2009; accepted Aug. 9, 2009

This work was supported by the Roche Foundation, The Wellcome Trust, and the Swiss National Science Foundation (Grant PPO0A-102721/1).

Correspondence should be addressed to Matthew E. Larkum, Department of Physiology, University of Bern, Bühlplatz 5, CH-3012 Bern, Switzerland. E-mail: larkum@pyl.unibe.ch.

B. Bathellier's present address: Institute of Molecular Pathology, Dr. Bohr-Gasse 7, A-1030 Vienna, Austria. D0I:10.1523/JNEUROSCI.1124-09.2009

Copyright $\odot 2009$ Society for Neuroscience $\quad$ 0270-6474/09/2912641-12\$15.00/0
}

tory bulb cells (Hasselmo et al., 1990; Brody and Hopfield, 2003; Margrie and Schaefer, 2003). In these models, the response of the cell is always supposed to be independent of the location of coactivated synapses on the dendritic tree. But alternatively, pyramidal cells may require the coordinated activity of specific clusters of synapses onto common dendritic compartments endowed with nonlinear dynamics (Mel, 1993; Larkum and Nevian, 2008). Such an architecture would certainly have a strong impact on the computational properties of piriform cortex (Poirazi and Mel, 2001; Rhodes, 2008) and sensory network design in general. A recent in vitro study indicates that the strength of olfactory bulb inputs onto piriform cortical pyramidal cells is rather variable but that the strongest connections are capable of generating pyramidal cell output (Franks and Isaacson, 2006). The potency of such inputs may be determined by the number and pattern of terminal synapses from individual axonal fibers onto the dendritic tree. Alternatively, these unusually strong connections may arise from the suprathreshold dendritic activity resulting from colocalized synaptic inputs (Polsky et al., 2004).

Here we use simultaneous dendritic and somatic patch-clamp recordings, together with extracellular minimal stimulation of LOT fibers and single cell simulations to investigate integration of olfactory synaptic inputs in principal neurons of the anterior piriform cortex.

\section{Materials and Methods}

Slice preparation. Anterior piriform cortex parasagittal brain slices $300-$ $350 \mu \mathrm{m}$ thick were prepared from 25 - to 35-d-old Wistar rats. Slices were cut in ice-cold extracellular solution (ACSF), incubated at $34^{\circ} \mathrm{C}$ for 20 min and stored at room temperature. During experiments, slices were superfused with in ACSF at $\sim 34^{\circ} \mathrm{C}$. The ACSF contained (in mM) 125 $\mathrm{NaCl}, 25 \mathrm{NaHCO}_{3}, 25$ glucose, $3 \mathrm{KCl}, 1.25 \mathrm{NaH}_{2} \mathrm{PO}_{4}, 2 \mathrm{CaCl}_{2}$, and 1 $\mathrm{MgCl}_{2}, \mathrm{pH} 7.4$, and was continuously bubbled with $5 \% \mathrm{CO}_{2} / 95 \% \mathrm{O}_{2}$. 

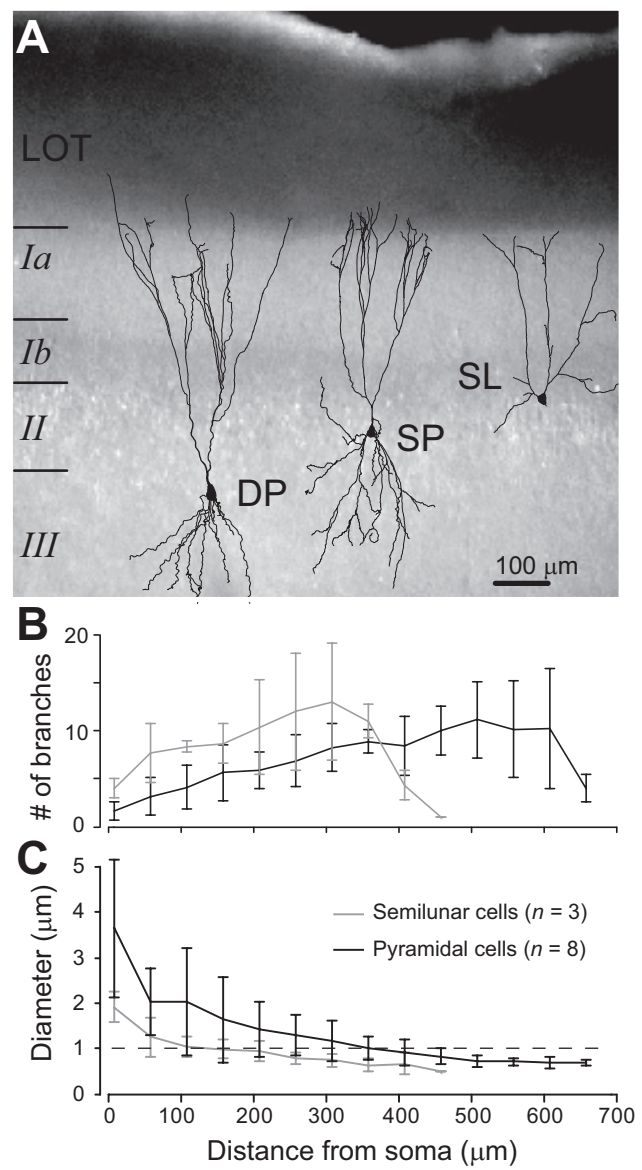

Figure 1. Dendritic architecture of piriform cortex excitatory neurons. A, IR-DIC image ( $4 \times$ magnification) of an anterior piriform cortex slice. Reconstructions of the dendrites of the three main excitatory cell types are superimposed (DP, deep pyramidal cell; SP superficial pyramidal cell; SL, semilunar cell). $\boldsymbol{B}$, Mean number of apical dendritic sub-branches versus distance from soma (error bar $=$ SD). $C$, Mean dendritic diameter versus distance from soma (error bar $=$ SD).

The intracellular solution contained (in $\mathrm{mm}$ ) $115 \mathrm{~K}^{+}$-gluconate, $20 \mathrm{KCl}$, 2 Mg-ATP, $2 \mathrm{Na}_{2}$-ATP, $10 \mathrm{Na}_{2}$-phosphocreatine, 0.3 GTP, 10 HEPES, 0.1 Oregon Green 488 BAPTA-1 (OGB-1), 0.01 Alexa 594, and biocytin $(0.2 \%), \mathrm{pH} 7.2$. Bicuculline methiodide $(1 \mu \mathrm{M})$ was added to the ACSF in focal stimulation experiments to try to lower the threshold for NMDA spikes.

Electrophysiology. Recording electrodes were made from thick-walled $(0.25 \mathrm{~mm})$ borosilicate glass capillaries and used without further modification. Dual whole-cell voltage recordings were performed from the soma (5-10 M $\Omega$ ) and dendrites (30-50 M $\Omega$ ) using Dagan BVC-700A amplifiers (Dagan Corporation). Data were acquired with an ITC-16 board (Instrutech) at $10 \mathrm{kHz}$ driven by routines written in the Igor software (Wavemetrics).

The small electrodes necessary for patching very thin basal dendrites resulted in high series resistances $\left(R_{\mathrm{s}}, 40-100 \mathrm{M} \Omega\right)$. Because the pipette behaves as a low-pass filter with a cutoff frequency inversely proportional both to $R_{\mathrm{s}}$ and to the pipette capacitance $\left(C_{\mathrm{p}}\right)$, a high $R_{\mathrm{s}}$ requires that $C_{\mathrm{p}}$ be carefully compensated so that high frequencies of the dendritic voltage are minimally distorted (Zhou et al., 2008). However, in another study using small pipettes, we compared small with normal-sized $(\sim 5 \mathrm{M} \Omega)$ pipettes at the cell body and found $\sim 5 \%$ error when measuring the somatic action potential (AP) amplitude with the smaller electrode (Nevian et al., 2007). Thus compensation can be done accurately even with high resistance pipettes.

LOT stimulation was performed with monopolar glass electrode (patch pipette, 5-10 M $\Omega, 0.1-100 \mathrm{~V}$ ) and an isolated voltage source delivering $100 \mu$ s pulses. Focal electrical extracellular stimulation was performed via a theta-glass pipette (5-10 M $\Omega$, bipolar, $100 \mu$ s, $0.1-100 \mathrm{~V}$ ).
Data analysis. Data analysis was performed using Matlab (The MathWorks). To describe the electrical properties of piriform pyramidal neurons we used two-port network theory as by Carnevale and Johnston (1982), which takes advantage of the fact that the spread of electrical signals in a neuron is best described in terms of the efficacy of signal transfer. In experiments where a long step of current of amplitude $I$ was injected at a single recording point [soma (s) or dendrite (d)], four voltage measurements can be defined: $V_{\mathrm{ss}}, V_{\mathrm{dd}}, V_{\mathrm{sd}}$, and $V_{\mathrm{ds}}$, where the first index indicates the recording site and the second the injecting site. Because of their linearity, the steady-state current/voltage relationships could be summarized with only three parameters: the two input resistances $\left(R_{\text {Input,soma }}=V_{\mathrm{ss}} / I\right.$ and $\left.R_{\text {Input,dend }}=V_{\mathrm{dd}} / I\right)$ at soma and dendrite measured from the slope of the voltage/current relationship at the site of current injection and the transfer resistance $\left(R_{\text {Trans }}=V_{\text {sd }} / I=V_{d s} / I\right)$ measured from the slope at the noninjecting site. For a perfectly linear circuit, $R_{\text {Trans }}$ is the same whether it is computed from somatic or dendritic current injections. Thus the symmetry ("reciprocity") of both measures provides a further evaluation of linearity and recording stability. Voltage transmission, or steady-state voltage attenuation, between dendrite and soma was computed as $V_{\mathrm{sd}} / V_{\mathrm{dd}}=R_{\mathrm{Trans}} / R_{\text {Input,dend. }}$ Current transmission is the ratio of the current arriving at the soma to the current I injected in the dendrite. According to Ohm's law, the current arriving at the soma is equal to the voltage generated at the soma divided by the somatic input resistance $V_{\mathrm{sd}} / R_{\text {Input,soma }}$. Hence, current transmission is $V_{\text {sd }} /\left(R_{\text {Input,soma }} I\right)=V_{\text {sd }} / V_{\text {ss }}=R_{\text {Trans }} / R_{\text {Input,soma }}$.

Local spontaneous unitary EPSPs were detected at the dendritic site and selected according to kinetic parameters with rise times of $<0.5 \mathrm{~ms}$. Peak amplitudes at soma and dendrites were measured using the average waveform of all detected EPSPs. EPSP attenuation ratio was defined as the ratio of the peak amplitude at the dendritic recording site to the peak amplitude at the somatic recording site. For injected EPSPs, current transmission was computed as $\int V_{\text {sd }}(t) d t /\left(R_{\text {Input,soma }} \int I(t) d t R_{\text {Input,soma }}\right)$ according to Rinzel and Rall (1974).

Spike amplitudes were measured as the difference between peak voltage and voltage immediately before spike initiation. Net dendritic spikes were obtained as the difference between actual voltage traces and the EPSP predicted from mean waveform in the subthreshold regime (all EPSPs with peaks below $-30 \mathrm{mV}$ ) scaled by the injected current. Results are given as mean $\pm \mathrm{SD}$.

To provide a quantitative evaluation of the variation of measured quantities with respect to the distance between somatic and dendritic recording site, we used an exponential fit, $f(x)=\exp \left(-x / \lambda_{\text {eff }}\right)$. The constant $\lambda_{\text {eff }}$ is termed "effective length constant." It should, however, not be confused with the canonical electrotonic length constant that appears in the cable equation.

Imaging. Neurons were filled via the somatic electrode with the calcium-sensitive dye Oregon Green BAPTA-1 (OGB-1, $100 \mu \mathrm{M}$, Invitrogen) and the calcium-insensitive dye Alexa 594 (Invitrogen). Dendritic recordings were made at least $15 \mathrm{~min}$ after the soma was patched. High-resolution images of small portions of the dendritic tree were acquired with a $60 \times$ objective and a CCD camera. Calcium-transients are reported as the mean change in OGB-1 fluorescence over a $1 \mathrm{~s}$ window normalized to the resting OGB fluorescence $\left(\left\langle\left(F(t)-F_{0}\right) / F_{0}\right\rangle\right)$. This measure is used instead of a peak measurement to remove linear summation effects and thereby emphasize nonlinear effects. A high-pass filter (Gaussian, $\sigma=20 \mu \mathrm{m}$ ) was applied on all images to remove background illumination and diffused light.

Computer simulations. Computer simulations were performed on biocytinfilled and Neurolucida-reconstructed experimental cells. Multicompartmental (33-107 dendrites) models were simulated in the NEURON platform. Each dendrite of length $L$ was divided into $N$ segments, where $N$ is the first integer number larger than $L / 8 \mu \mathrm{m}$. For each cell, we first determined the values of homogeneous membrane resistance $R_{\mathrm{m}}$ and axial resistance $R_{\mathrm{a}}$ that produced the best fit to experimentally measured input and transfer resistances. The average values for $R_{\mathrm{a}}$ and $R_{\mathrm{m}}$ were $173 \pm 101 \Omega \cdot \mathrm{cm}$ and $23 \pm 7 \mathrm{k} \Omega \cdot \mathrm{cm}^{2}$, respectively. Then, the membrane capacitance was optimized to best fit the transient phase of step responses $\left(C_{\mathrm{m}}=1.3 \pm 0.3 \mu \mathrm{F} \cdot \mathrm{cm}^{-2}\right)$. For unitary EPSP simulations, AMPA synapses were modeled with $0.2 \mathrm{~ms}$ rise time, decay time constant 
A

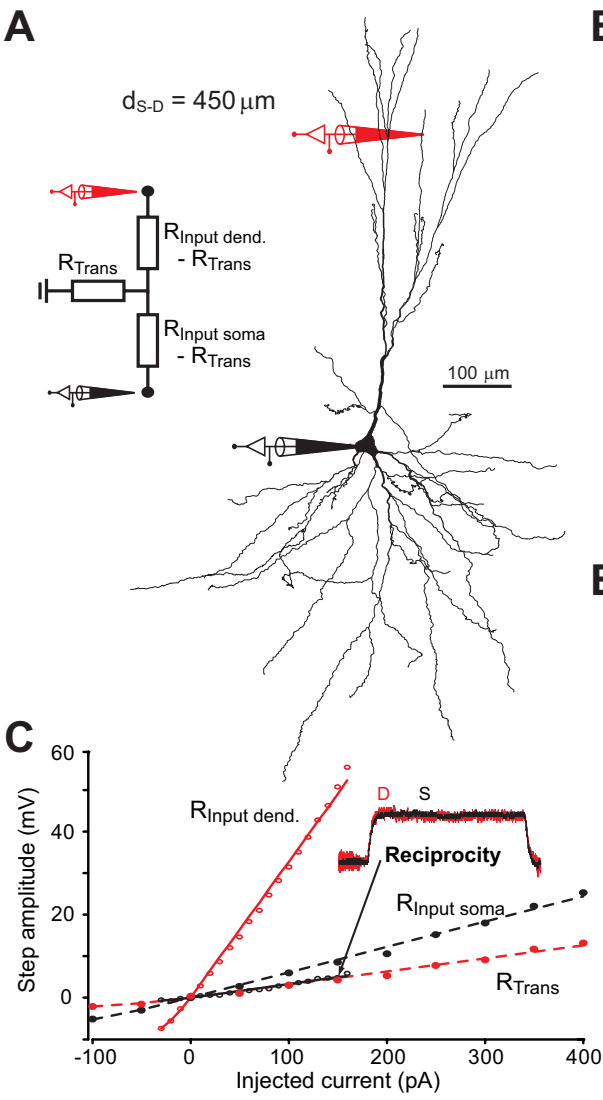

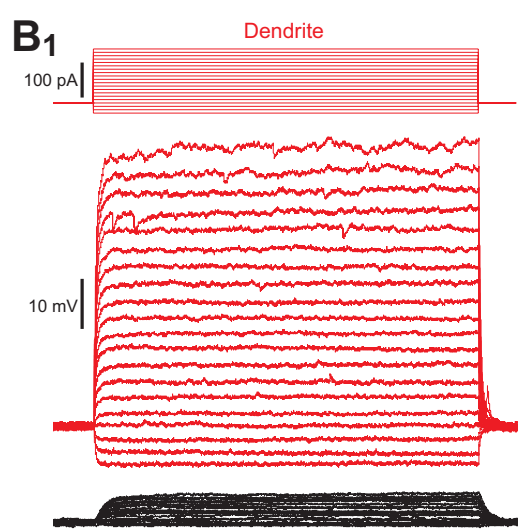
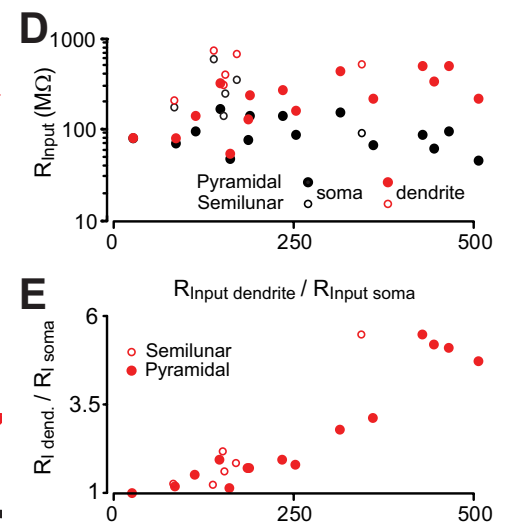

$\mathbf{B}_{2}$

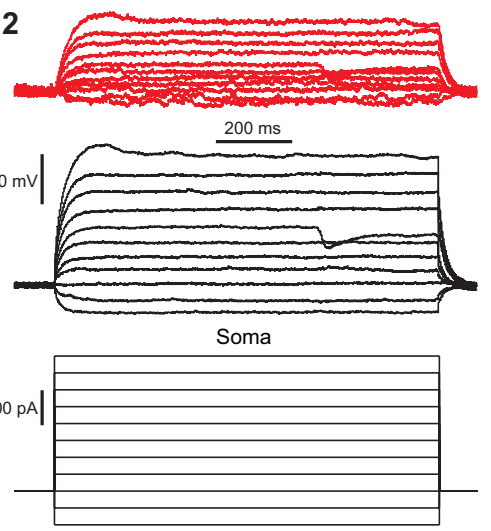

$\mathbf{F}$

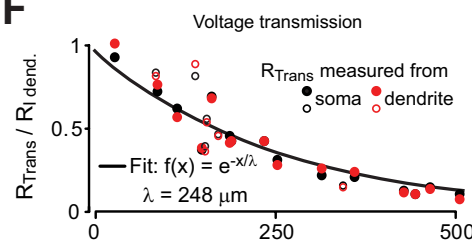

G

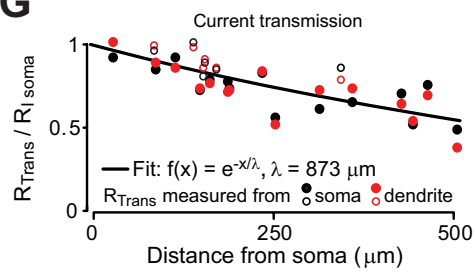

Figure 2. Steady-state properties of passive electrical conduction. $A$, Right, Reconstruction of a piriform cortex (PC) pyramidal cell with the positions of somatic and dendritic $\left(d_{\mathrm{S}-\mathrm{D}}=450 \mu \mathrm{m}\right.$ from soma) recordings. Left, Two-port T-circuit corresponding to the recording setup. B, Top, Step current injections to the dendrite and resulting voltage response at dendrite (red) and soma (black). Bottom, Same as top but for injections at the soma. C, Measured steady-state voltage (circles) in soma and dendrite as a function of injected current, the slope of linear regressions (lines) gives input or transfer resistances. The transfer resistanceis the same for both directions of currentinjection (reciprocity) showing the linearity of electrical conduction in the dendrites. D, Absolute value of input resistances at the dendrite (red) and soma (black) for all paired recording plotted as a function of the distance between the recording sites (i.e., each point is a different recording in a different cell). Filled symbols: pyramidal cells; empty symbols: semilunar cells. $\boldsymbol{E}$, Ratio of dendritic and somatic input resistances. $\boldsymbol{F}$, Ratio of transfer resistance and dendritic input resistance (i.e., fraction of transmitted voltage from dendrite to soma). $\boldsymbol{G}$, Ratio of transfer resistance and somatic input resistance (i.e., fraction of transmitted current from dendrite to soma, or equivalently, fraction of transmitted voltage from soma to dendrite).

of $1 \mathrm{~ms}$, peak conductance of $1 \mathrm{nS}$, and reversal potential of $0 \mathrm{mV}$. Simulated voltage-clamp experiments showed that such synapses gave on average a peak current at soma of $10.5 \pm 5.6 \mathrm{pA}$, very close to the quantal size of 12 pA measured by Franks and Isaacson (2006).

Statistical analysis of single-fiber experiments. To identify LOT singlefiber inputs, stimulation strength (voltage) was slowly increased while EPSP amplitude at the soma was monitored. If only few marked steps in EPSP amplitude were observed, and if EPSP amplitude in between steps did not increase slowly with stimulation strength, the steps were interpreted as the successive recruitment of unitary LOT fibers (see Fig. 9). To detect whether single-fiber EPSP resulted from spatially distributed or clustered synaptic inputs (see Fig. $9 E, F$ and last paragraph of the Results), we used the following reasoning. For a single isolated releasing synapse, the ratio of EPSP amplitudes measured at the soma and at a randomly chosen dendrite depends strongly on the respective positions of the dendritic recording pipette and of the synapse. Hence, we reasoned that if all synapses of a single fiber are clustered onto one dendritic location, one would expect the ratio of the dendritic to somatic EPSP to stay constant from trial to trial, whereas if the input is distributed this ratio will fluctuate depending on which other synapses are activated on any one trial. The only caveat for this assumption is that if none of the inputs (or very few) are on the recorded dendrite then the input might still be distributed but not fluctuate. To control for this case, we examined only experiments where the input was larger at the dendritic than at the somatic recording.

In mathematical terms, we described the amplitude of EPSPs at the soma and at the dendrite after step number $p$ by two random variables $S_{p}$ and $D_{p}\left(S_{0}\right.$ and $D_{0}$ are for baseline noise). If we suppose that each step corresponds to the activation of a new axonal fiber whose EPSP adds up linearly with the ones from preceding fibers, we can write $S_{p} \equiv F_{p}+S_{p-1}$ and $D_{p} \equiv \alpha_{p} F_{p}+D_{p-1}$, where $F_{p}$ is the somatic amplitude of the EPSP resulting from fiber $p$ and $\alpha_{p}$ is the ratio of dendritic to somatic EPSP amplitude. If synapses of fiber $p$ are clustered, then $\alpha_{p}$ is constant and equal to $\left(\left\langle D_{p}\right\rangle-\left\langle D_{p-1}\right\rangle\right) /\left(\left\langle S_{p}\right\rangle-\left\langle S_{p-1}\right\rangle\right)$. We also expect from the linear summation of successive fibers that the random variables $D_{p}-\alpha_{p} S_{p}$ (dendritic amplitude minus prediction from somatic amplitude) and $D_{p-1}-\alpha_{p} S_{p-1}$ (activity of preceding step viewed as a background noise) have the same underlying statistical distribution. This could be evaluated with a Kolmogorov-Smirnov test for the identity of two distributions. Any significant deviation from the null hypothesis implies that $\alpha_{p}$ is not constant and therefore that the EPSP of fiber $p$ was generated by randomly activated synapses distributed over different places of the dendritic tree. If no significant deviation is found, it means either that all synapses were clustered or that all synapses were distributed with respect to the dendritic recording site in such a way that they individually showed similar ratios of dendritic to somatic EPSP amplitudes. The latter case can easily occur when all synapses are so distant from the dendritic electrode that they all exhibit attenuation ratios close to 1 . Hence, dual recordings of single-fiber EPSP in which the observed attenuation ratio stays close to 1 turn out to be inappropriate for this analysis.

\section{Results}

Basic electrotonic properties

Whole-cell dual dendritic and somatic patch-clamp recordings were made from principal neurons of the anterior piriform cor- 
tex in young adult rats (P27-P35). The anterior piriform cortex was chosen because it is a primary olfactory cortex and can be prepared in parasagittal slices where the laminar structure and the lateral olfactory tract are readily identifiable (Franks and Isaacson, 2006; Suzuki and Bekkers, 2006) (Fig. 1A) permitting reliable stimulation of afferent fibers. We investigated three classically defined subtypes of excitatory neurons (Fig. 1A): superficial and deep pyramidal cells $(n=$ 26) and semilunar cells (SL, $n=7$ ) based on the location of their cell bodies in upper layer 2 and their lack of basal dendrites (Suzuki and Bekkers, 2006). Since the boundary between layer 2 and 3 could not be reliably distinguished, we pooled the results of superficial and deep pyramidal neurons in this study. The apical dendrite of both pyramidal and semilunar neurons branched rapidly having in general already one or more bifurcations as close as $150 \mu \mathrm{m}$ from the soma (Fig. $1 B$ ). For semilunar cells, the dendritic diameter decreased rapidly to $<1 \mu \mathrm{m}$ at distances greater than $\sim 150 \mu \mathrm{m}$ from the soma. This was in contrast to pyramidal cell dendrites which maintained a diameter $>1$ $\mu \mathrm{m}$ up to $\sim 400 \mu \mathrm{m}$ from the cell body (Fig. $1 C$ ). For both cell types, branches quickly became difficult to visualize under infrared differential contrast (IR-DIC) beyond the main bifurcation. However, including the red fluorescent dye Alexa 594 in the somatic patch pipette enabled us to identify dendritic branches well into layer 1 using a semiautomated visualization program. With this method we were able to perform dendritic recordings from all parts of the apical dendrites up to the boundary between layer Ia and the lateral olfactory tract (Fig. 2A). Cells were also filled with biocytin in the pipette solution and some were later reconstructed for compartmental modeling.

Long, stepwise current injections (1000 ms) were used to determine the steady-state properties of the dendrites (Fig. 2B). Around resting voltage, there was no sag in the responses to long current injection either in the dendrite or at the cell body (Fig. $2 B$ ) indicating little or no $I_{\mathrm{h}}$ conductance at physiological membrane potentials (Harris and Constanti, 1995; Sciancalepore and Constanti, 1998). We also observed that at all recording sites the current/voltage relationship was nearly linear for depolarized subthreshold voltages (Fig. 2C). Furthermore, the response to current injection was always identical at the reciprocal recording location ("reciprocity") (Fig. 2C, inset). Electrical conduction between the 2 electrodes could therefore be well described by a linear two-port circuit (Fig. 1 A, inset) (Carnevale and Johnston, 1982). As expected from cable theory of branching neurons (Rall and Rinzel, 1973), input resistance was lower in the soma than in the dendrites (Fig. 2D), meaning that a current injected in the dendrite leads to a greater local voltage change than if injected in the soma (Fig. 2C). Note that recordings at very large distances from the soma $(>300 \mu \mathrm{m})$ could only be achieved in large pyramidal cells (somatic input resistance $<100 \mathrm{M} \Omega$ ). The ratio of dendritic to somatic $R_{\text {Input }}$ increased as a function of distance from the soma in both cell types and could reach values up to 6 at distal locations (Fig. 2E). In both cell types, voltage transmission

Figure 3. Passive conduction of synaptic transients. $\boldsymbol{A}$, Left, Examples of spontaneous EPSPs recorded simultaneously in a

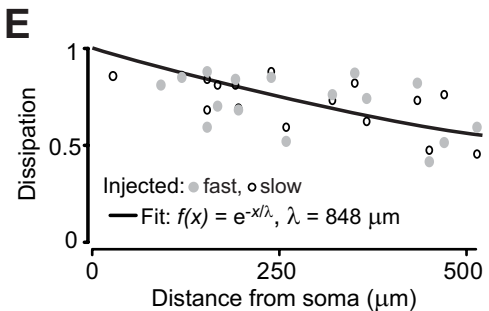

Distance from soma $(\mu \mathrm{m})$

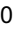

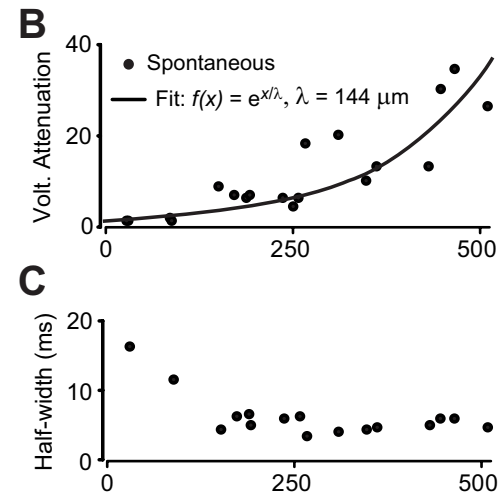
Injected EPSPs
$\mathrm{d}_{\mathrm{S}-\mathrm{D}}=240 \mu \mathrm{m}$

Soma Dendrite

ast
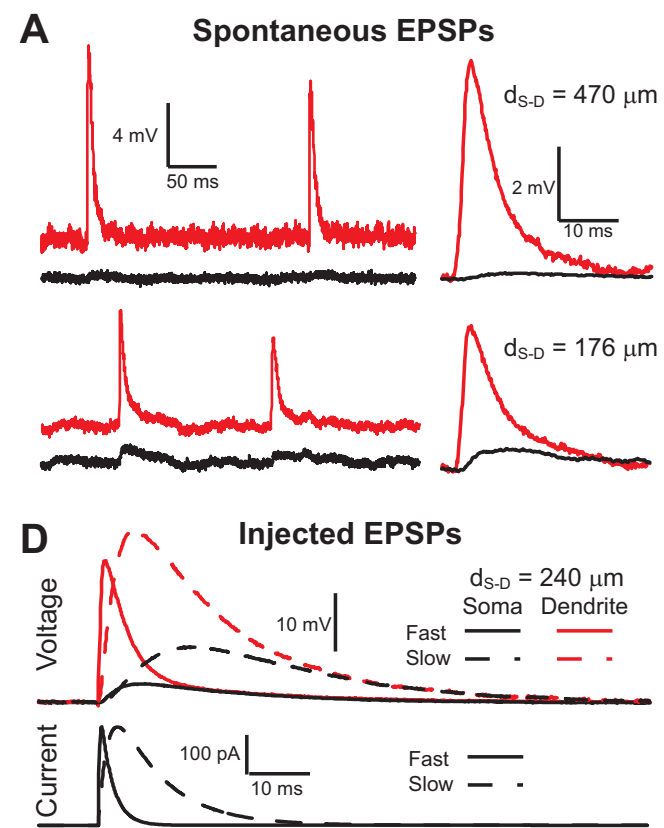
dendrite and at the soma of two neurons. Right, Average of all recorded spontaneous EPSPs for each neuron. $\boldsymbol{B}$, Attenuation of current transients injected in the dendrite to mimic an EPSC. Top, Corresponding voltage response in the dendrite (red) and in the soma (black). $\boldsymbol{E}$, Fraction of the total injected charge reaching the soma for fast and slow EPSPS.

along the dendrites was inefficient. The ratio of somatic to dendritic voltage could be fitted with a decaying exponential giving a length constant of $\lambda_{\text {eff }}=248 \mu \mathrm{m}$ (Fig. $2 F$ ). However, current transmission computed as the ratio of the current arriving at the soma to the current injected in the dendrite (see Materials and Methods) was relatively efficient $\left(\lambda_{\text {eff }}=873 \mu \mathrm{m}\right)$ (Fig. $2 G$ ), meaning that the large difference of voltage between dendrite and soma was explained by the large difference of their input resistance (which reflects their relative size) rather than by a large loss of current in the dendritic tree.

\section{Synaptic events and propagation of transient signals}

The peak amplitude of local spontaneous EPSPs detected at the dendrite was largely attenuated at the soma (up to 35 times) (Fig. $3 A, B)\left(\lambda_{\text {eff }}=144 \mu \mathrm{m}\right)$ as observed in other thin dendrites (Nevian et al., 2007; Larkum et al., 2009). The half-width of these spontaneous EPSPs was also much shorter at the dendrite than at the soma ( $4.6 \pm 0.8 \mathrm{~ms}$ vs $22.6 \pm 8.0 \mathrm{~ms})$ but relatively constant as a function of recording distance from the soma (Fig. $3 C$ ). To estimate the electric charge dissipation for transient synaptic events in these dendrites, we injected EPSC-like waveforms (short and long with rise times 0.2 and $2 \mathrm{~ms}$; decay times 1 and 8 ms) (Fig. 3D). Because charge is the time integral of current, it is expected that in a linear circuit the efficiency of charge propagation is independent of EPSC waveform and equals forward propagation of steady-state currents (Rinzel and Rall, 1974; Carnevale and Johnston, 1982). Indeed, the amount of charge that entered the cell at the site of current injection showed minimal dissipation from the dendrite to the soma and the effective length constant was close to the one observed for current transmission $\left(\lambda_{\text {eff }}\right.$ $=848 \mu \mathrm{m}$ for charge transmission) (Fig. $3 E$ ). Therefore, the dramatic voltage attenuation of transient synaptic inputs most likely reflects the high input impedance of distal dendrites as well as transient charge accumulation effects (Rinzel and Rall, 1974; Carnevale and Johnston, 1982), rather than loss of current. 

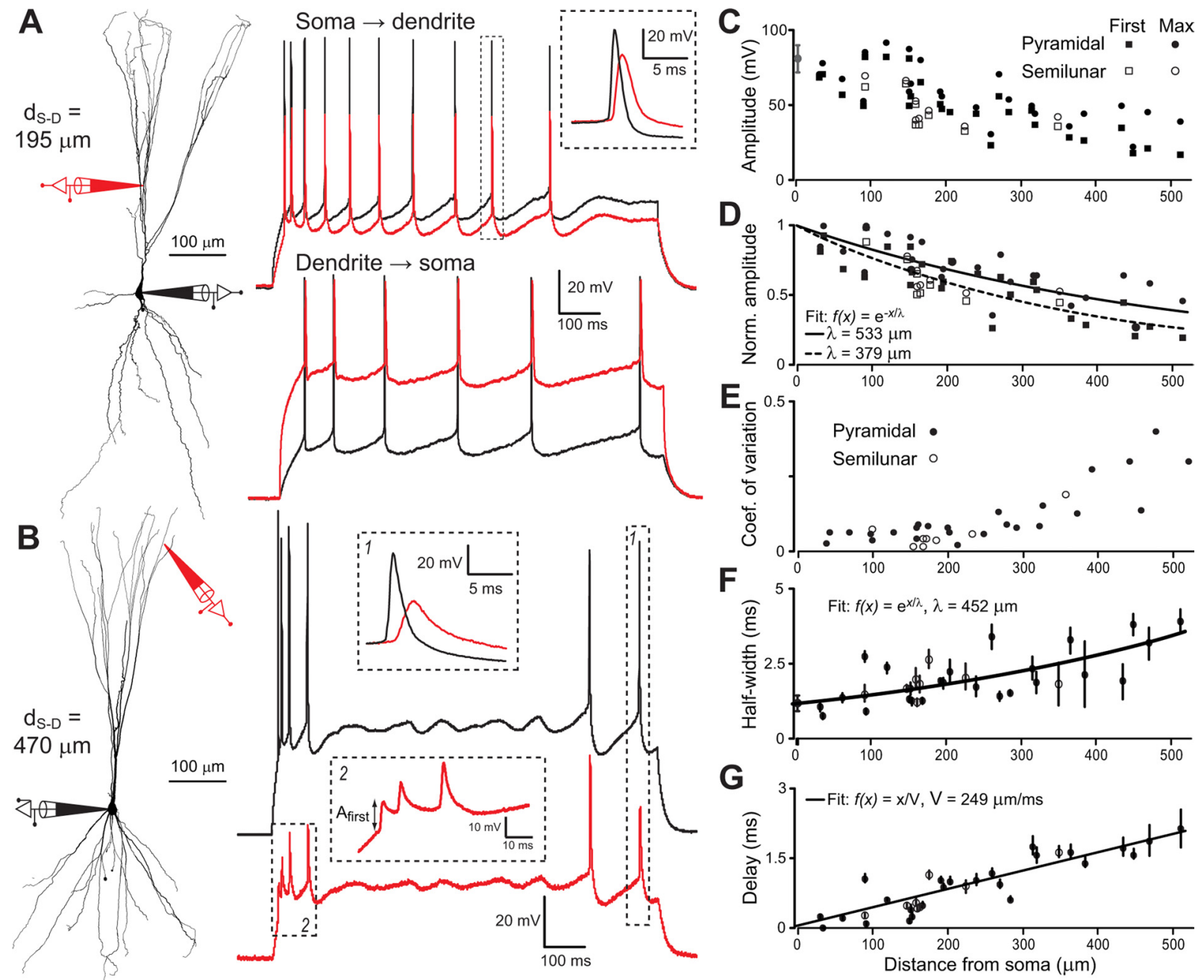

Figure 4. Action potential backpropagation. $A$, Left, Reconstruction of a PC pyramidal cell with the locations of somatic and dendritic $\left(d_{S-D}=195 \mu m\right.$ from soma) recordings. Right, Spike trains elicited by step current injections recorded at the somatic and dendritic locations. The inset shows a magnification of the spike enclosed in the dashed rectangle. $\boldsymbol{B}$, Same as $\boldsymbol{A}$ (top) for a distal dendritic ( $d_{S-D}=470 \mu \mathrm{m}$ from soma) recording and step current at the soma. Note the variability of backpropagating spike amplitude in the dendritic recording. $C$, Backpropagating spike amplitude in the dendrite as function of somatic distance: circles, maximum AP amplitude in the train; squares, first spike of a train. Recordings in semilunar cells are shown with empty markers. $\boldsymbol{D}$, Ratio of backpropagating spike amplitude to somatic spike amplitude: circles, maximum in each train; squares, first spike of a train. $\boldsymbol{E}$, Coefficient of variation of backpropagating spike amplitude computed over all recorded spikes in a cell. $\boldsymbol{F}$, Half-width of the backpropagating spike (average and SD). G, Peak-to-peak delay between somatic and backpropagating dendritic spikes (average and SD).

\section{Backpropagating APs}

Actively backpropagating APs have been shown to be important in many neurons to signal the activity of the neuron to synapses (Magee and Johnston, 1997; Markram et al., 1997; Bi and Poo, 1998; Häusser et al., 2001; Froemke et al., 2005; Sjöström and Häusser, 2006; Kampa et al., 2007). We examined backpropagation in piriform pyramidal neurons with suprathreshold step current injections evoking trains of APs at the soma while recording in the dendrite. We observed rapid frequency adaptation of spike trains at the soma in all pyramidal neurons (Fig. $4 A, B$ ) but not in semilunar cells (data not shown) as demonstrated previously (Suzuki and Bekkers, 2006).

Interestingly, the propagation along the dendrite of APs within a given train could be variable and this variability depended on distance from the soma. For very distal dendritic recordings, there was typically an increase in amplitude at the beginning of the AP train followed by strongly variable amplitudes (Fig. $4 B$ ). This phenomenon has never been observed in other mammalian pyramidal neurons but has been observed in the pyramidal neurons of the three-layered turtle cortex (Larkum et al., 2008). The maximum dendritic AP amplitude in the train decreased as a function of distance over all cells. Fitting the data with a decaying exponential yielded an effective length constant of, $\lambda_{\max }=553 \mu \mathrm{m}$ (Fig. $4 C, D$, circles) which is comparable to measurements performed in other cortical pyramidal neurons where active boosting is supported by dendritic $\mathrm{Na}^{+}$channels (Stuart and Sakmann, 1994; Stuart et al., 1997; Waters et al., 2003). On the other hand, the dendritic AP amplitude, $\lambda_{\text {first }}$, measured for the first AP of each train decreased more strongly with distance $\left(\lambda_{\text {first }}=319 \mu \mathrm{m}\right)$ (Fig. $4 C$, squares). The within-train variability of dendritic AP amplitude clearly increased as a function of distance from the soma especially after $300 \mu \mathrm{m}$ from soma (Fig. $4 E$ ). Other parameters such as half-width (Fig. $4 F$ ) and delay (from peak to peak) (Fig. $4 G$ ) also increased with distance. The decrease in maximal dendritic AP amplitude with distance from soma was not significantly different in semilunar cells and in pyramidal cells $(p=0.08$, Student's $t$ test for the deviation with respect to global fit).

\section{Calcium dynamics in the dendrites}

$\mathrm{Ca}^{2+}$ channels have been found in the dendrites of all neurons so far tested (Migliore and Shepherd, 2002). These channels are 

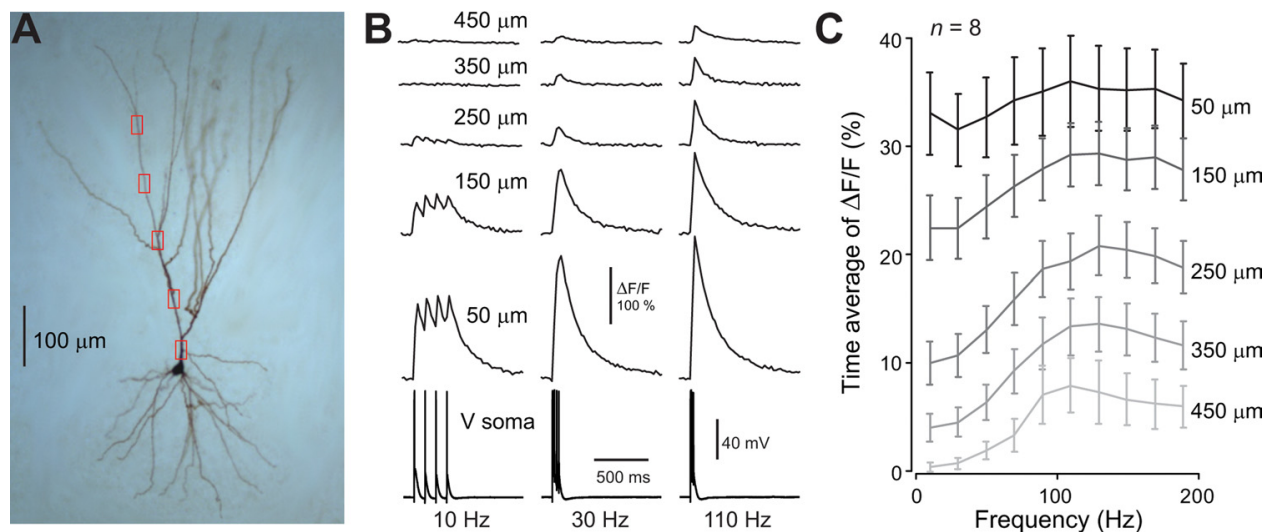

Figure 5. Calcium influx triggered by backpropagation of action potential bursts. $\boldsymbol{A}$, Biocytin-filled layer 3 pyramidal neuron. Red rectangles indicate imaging locations. $B$, Calcium responses (OGB-1) to trains of four action potentials $(10,30,110 \mathrm{~Hz})$ recorded at the five dendritic locations shown in $A$. Electrical recordings from the soma are shown at the bottom. C, Time-averaged calcium response measured over a $1 \mathrm{~s}$ poststimulus window as a function of spike train frequency at five different distances (average over $n=8$ neurons).

often activated by backpropagating APs. We were also able to detect calcium influx into the dendrites of olfactory cortex neurons following trains of backpropagating APs initiated by brief depolarizing current steps at the soma (Fig. 5) $(n=8)$. Strong calcium transients were observed for all frequencies of APs in a train in the proximal part of the dendritic tree (Fig. $5 B$ ). In the most distal regions, $\mathrm{Ca}^{2+}$ entry was a function of the AP-train frequency, being close to zero for $10 \mathrm{~Hz}$ (Fig. 5B,C). However, AP-triggered calcium entry in very distal dendrites also strongly depended on the resting voltage of the neuron. When constant current was injected to the soma so that the neuron could spontaneously fire, AP backpropagation was improved and even a single spike could trigger large calcium transients distally (supplemental Fig. 1, available at www.jneurosci.org as supplemental material). This suggests that calcium entry can be boosted by background activity as observed in neocortical pyramidal cells in vivo (Waters and Helmchen, 2004).

\section{Local dendritic spikes}

So far, our results show that the dendrites of olfactory cortical pyramidal neurons actively support backpropagating signals. But do these active properties also support forward propagation of incoming signals and thus account for the sometimes extremely large influence of LOT inputs observed at the soma (Franks and Isaacson, 2006)?

To investigate this question, we injected depolarizing current into the dendrites in the shape of an EPSC (Fig. 6). In many cells, there was a nonlinear threshold response to increasing current injection indicating local electrogenesis. This either took the form of a short spike followed by a somatic action potential $(n=$ 3 ; at more proximal locations) (Fig. 6A) or occurred without being associated to a somatic $\mathrm{AP}(n=12$; at more distal locations) (Fig. 6B). This short spike could be triggered both by short and long EPSCs (decay time of $1 \mathrm{~ms}$ vs $8 \mathrm{~ms}$ ), but was more distinguishable for long EPSCs (Fig. $6 A_{2}, A_{3}, B_{2}, B_{3}$ ). In all cases, the net active component (calculated by subtracting the linear summation of the subthreshold responses) involved a short, mostly allor-none component followed by a longer, linearly increasing negative component (Fig. $6 A_{4}, B_{4}$ ). Dendritic spikes were not seen within $150 \mu \mathrm{m}$ from the soma. In this region current injection resulted in a somatic AP that propagated back to the dendritic location (Fig. 6C). Dendritic spikes were followed by somatic spikes only between 150 and $220 \mu \mathrm{m}$ from the soma. For larger distances, EPSP injections were inefficient in driving the neuron to fire despite the occurrence of the dendritic spike. As in other thin dendrites (e.g., Nevian et al., 2007), attenuation of forward-propagating spikes $(18.8 \pm 15.1)$ was much larger than attenuation of backpropagating APs $(1.6 \pm 0.5)$. The resulting net subthreshold depolarization at the soma was never larger than $2 \mathrm{mV}$ (Fig. $6 A_{4}$ ), and in most cases negligible (especially for dendritic spikes generated distally, mean amplitude for distances $>250 \mu \mathrm{m}: 0.22 \pm 0.20 \mathrm{mV}, n=8$ ) (e.g., Fig. $6 B_{4}$ ). Importantly, the hyperpolarizing component dominated the active response. In most cases, the time integral of the net dendritic events decreased linearly with increasing injected charge (Fig. 6D). Hence, these dendritic events induced a loss of current (probably due to opened active conductances), rather than a boost. This loss could represent up to $75 \%$ of the charge that would reach the soma in the absence of the dendritic event (Fig. 6E). Therefore, the observed dendritic spikes most likely contribute little to somatic activity, and result mainly in sublinear transmission of dendritic inputs.

\section{Response to focal stimulation}

Another mechanism that can generate large all-or-none responses in dendrites is the NMDA spike (Schiller et al., 2000) that is thought to result from simultaneous activation of colocalized synapses in thin dendrites. We stimulated extracellularly with a theta-glass electrode close to the dendritic recording site (e.g., Fig. $7 A)(n=17)$ as recently described in neocortical pyramidal neurons (Nevian et al., 2007). While increasing stimulation strength, we found that sometimes huge local input could be produced (up to $80 \mathrm{mV}$ at the dendrite) (e.g., Fig. $7 B-D$ ). The attenuation ratio of this input tended to decrease slightly with increasing stimulation strength (Fig. $7 B$, inset), indicating that the input became more distributed along the dendritic tree. However, despite the large local depolarization achieved, we never observed any plateau depolarization indicative of a local NMDA spike at any input frequency tested $(20-200 \mathrm{~Hz})$. After applying the NMDA receptor blocker D-AP5 (50 $\mu \mathrm{M}, n=3)$ during focal stimulation we observed very little decrease in the amplitude of large EPSPs, but a reduction of their duration and integral, both at the soma and dendrite [e.g., Fig. 7C (inset),F]. Therefore, NMDA receptors were activated by local stimulation, probably unblocked by the large depolarization, but did not elicit typical NMDA spikes. Because they are regenerative events, NMDA spikes result in abrupt changes of EPSP amplitude when stimulation strength is increased. This can even be observed at the soma. In some cases, we observed such steps in the stimulus/response (Fig. 7G). However, when tested 

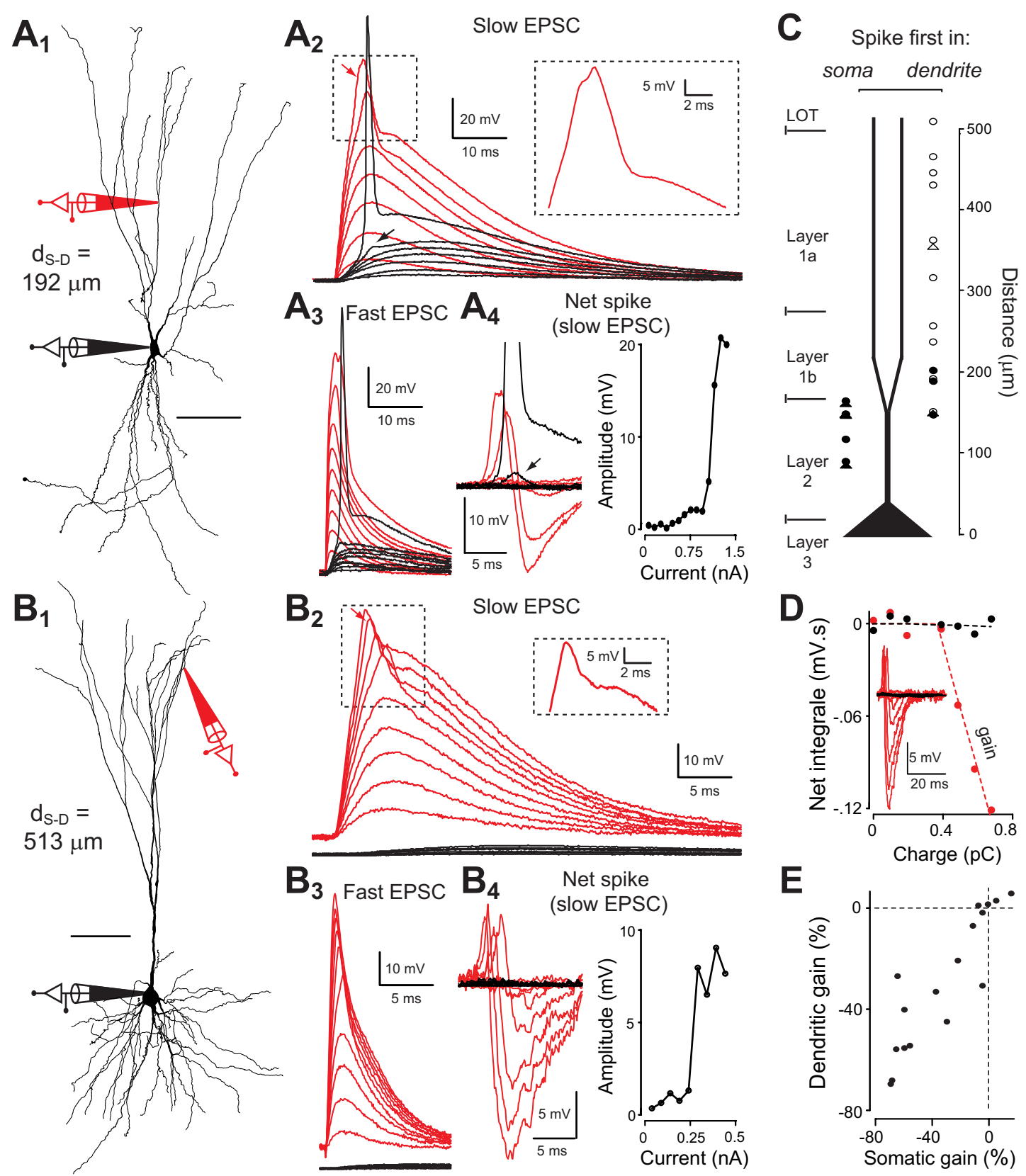

Figure 6. Generation of small local dendritic spikes. $A_{1}$, Reconstruction of a PC pyramidal cell with the locations of somatic and dendritic $\left(d_{S-D}=192 \mu \mathrm{m}\right.$ from soma) recordings shown schematically. $A_{2}$, Voltage at soma and dendrite resulting from increasing EPSC-like injections in the dendrite. Large dendritic depolarizations generate a fast sodium spike (see magnification in the inset of the spike indicated by the upper arrow). The lower arrow shows the effect of the dendritic spike when it failed to trigger an AP in the axon hillock. $A_{3}$, Same as $A_{2}$ but for a faster EPSC. $A_{4}$, Left, Traces of net spikes in the soma and dendrite (actual voltage minus voltage predicted from subthreshold EPSPs) for $A_{2}$. Right, Net spike amplitude versus injected current amplitude for $A_{2}$. $\boldsymbol{B}$, Same as $\boldsymbol{A}$ for a more distal recording $\left(d_{s-D}=513 \mu \mathrm{m}\right.$ from soma). In this case, it was not possible to trigger a somatic AP. C, Plot showing whether a spike was initiated first in the dendrite (right) or in the soma (left) for an increasing current injection at the dendrite. Circles indicate pyramidal cells and triangles semilunar cells. Empty markers indicate when no somatic spike could be triggered. $\boldsymbol{D}$, Integral of the total net spike (as showed in the inset), at the soma (black) and at the dendrite (red), plotted as a function of injected charge for the example shown in $\boldsymbol{B}$. The red and black dashed lines represents fits of the data with a piecewise linear function $\left(f(x)=0\right.$ if $x<x_{0}$ and $f(x)=\alpha\left(x-x_{0}\right)$ if $\left.x \geq x_{0}\right)$. The fitted parameters $\alpha$ and $x_{0}$ are, respectively, the voltage gain and the current threshold for dendritic spikes. $E$, Voltage gains (fitted $\alpha$ expressed in percentage of the dendritic input resistance) in soma and dendrite for all observed dendritic spikes ( $n=15$ ).

systematically with bath application of D-AP5, the amplitude of these steps appeared independent of NMDA conductances (Fig. 7 H,I), indicating that they do not result from NMDA spikes. The most probable explanation for these steps might be a nongradual recruitment of incoming fibers.

Simulation of synaptic integration in reconstructed neurons Sensory inputs from the olfactory bulb contact pyramidal cells on the distal part of their apical dendritic tree (Neville and Haberly,
2004). What connectivity patterns would be most appropriate to drive piriform cortex pyramidal cells? So far we have observed that local synaptic inputs readily generate large EPSPs in these dendrites with a small impact on the soma, and we have not found any regenerative process that could boost synaptic transmission to the soma (on the contrary, dendritic spikes had a net negative impact on current transmission). Previous studies have showed that, in these conditions, activation of nearby synapses leads to sublinear input summation because the resulting large 
voltage increase in the dendrite can strongly reduce the driving force of synapses (Rall, 1970; Margulis and Tang, 1998). Hence, clustered synaptic inputs are expected to be suboptimal in depolarizing the soma. However, because EPSP amplitude rapidly decreases in the dendrites with distance from the active synapse, spatially distributed inputs are expected to minimize sublinear summation and thereby to be more efficient in depolarizing the soma (Rall, 1970).

To evaluate the impact of different numbers of distributed synapses on the distal apical tree of piriform cortex pyramidal cells, we made compartmental models of reconstructed neurons in which the passive parameters (uniform membrane resistance, capacitance and axial resistance) were adjusted to fit the experimentally determined responses to long current pulses injected in the soma and in the dendrite (Fig. $8 A, B$ ). When a short EPSC (rise $0.2 \mathrm{~ms}$, decay $1 \mathrm{~ms}$ ) was injected at a single dendritic location, EPSP amplitude at the injection site was always larger than in the soma and the difference increased with distance reaching $>100$-fold for EPSCs injected at the tip of dendrites (Fig. $8 C$, each black dot represents a different injection site). The average $\lambda_{\text {eff }}$ for EPSP amplitude attenuation was $110 \pm 24 \mu \mathrm{m}$ ( $\mathrm{SD}, n=7$ ), a value slightly smaller than the one measured in experiments (cf. $\lambda_{\text {eff }}=144$ $\mu \mathrm{m})$, probably due to a bias in the experimental recordings toward the largest dendrites at very distal locations.

However, if instead of a single localized EPSC, we injected one EPSC at all points of the tree equidistant from the soma (i.e., same anatomical length of the dendritic path from the soma to the injection points), we observed, as expected, that the ratio between somatic and dendritic EPSP amplitudes was much reduced (Fig. 8C, red curve and gray shading). Interestingly, because the number of branches also increases with distance the ratio was stabilized between 2 and 3 in the region of the apical tree that receives inputs from the olfactory bulb (Fig. $8 C$, red curve from $\sim 200$ to $500 \mu \mathrm{m})$. Hence, the branching architecture of pyramidal neurons seems to be well matched to a distributed input connectivity (i.e., there are enough branches to compensate the increase in dendritic input resistance).

We simulated random distributions of 10, 25, and 100 synapses, modeled as AMPA conductances with the same kinetics as injected currents. The maximum single-channel conductance $(1 \mathrm{nS})$ was chosen to approximate quantal synaptic currents observed in pyramidal cells of the piriform cortex (see Materials and Methods). Twenty-

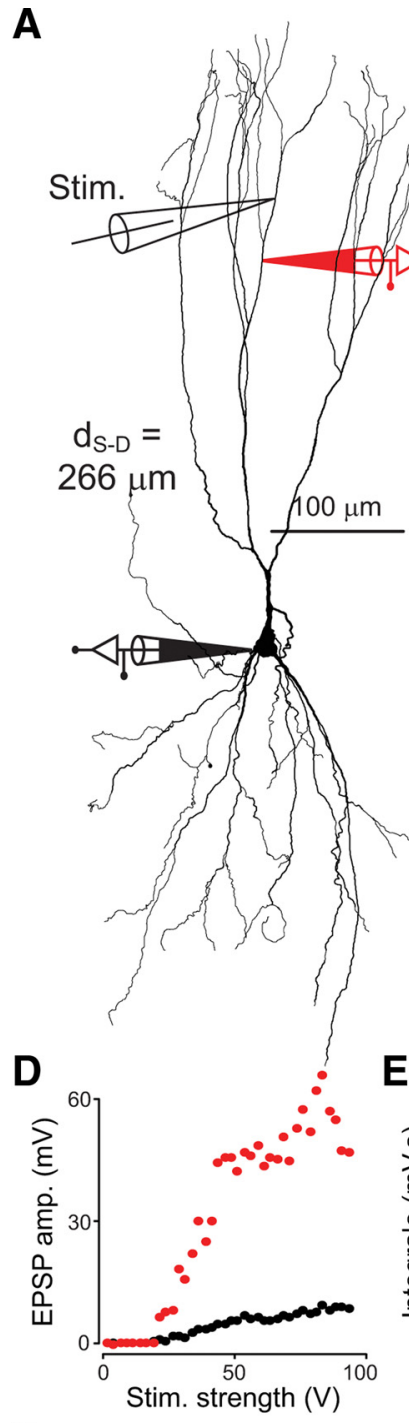

B $20 \mathrm{~Hz}$ Minimal stim.
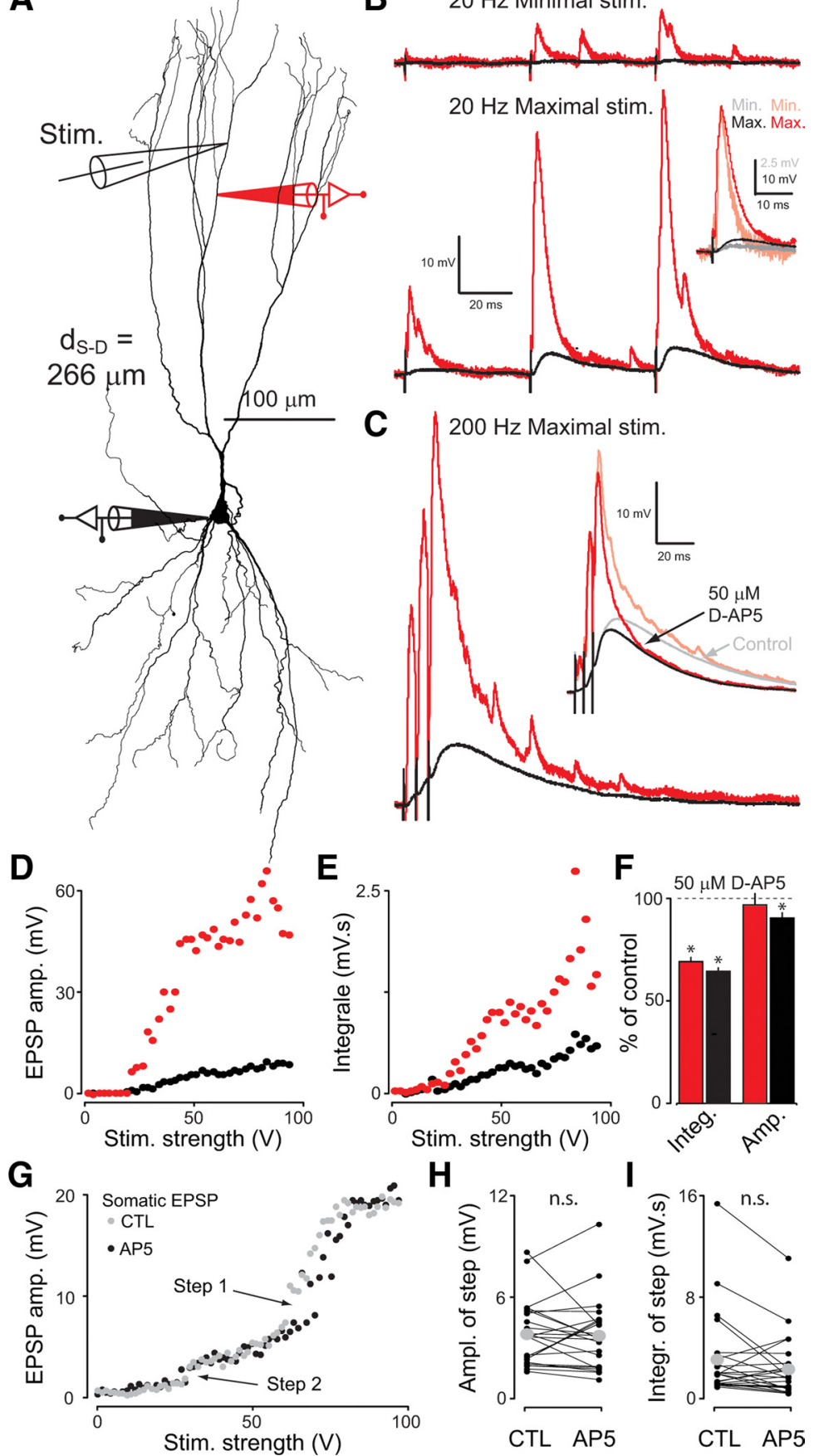

4
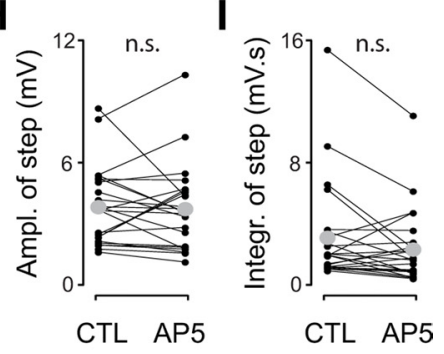

Figure 7. Focal extracellular stimulation. $A$, Reconstruction of a neuron with locations of dendritic (red, $d_{S-D}=266 \mu \mathrm{m}$ from soma) and somatic (black) recording sites. A third pipette was used for focal extracellular stimulation just above the dendritic recording. B, Minimal (top) and maximal (bottom) EPSPs generated by trains of three focal stimulations at $20 \mathrm{~Hz}$ (single sweeps are shown). The inset compares time course and attenuation of minimal (light red, shorter) and maximal (dark red, longer) dendritic EPSPs. C, EPSPs generated by three pulses stimulation at $200 \mathrm{~Hz}$. The inset compares the average EPSPs in the presence (black and red, shorter EPSPs) and absence (gray and light red, longer EPSPS) of the NMDA blocker D-AP5. D, EPSP amplitude in soma and dendrite as a function of stimulus strength for the $200 \mathrm{~Hz}$ stimulation. $\boldsymbol{E}$, Same as $\boldsymbol{D}$ but for the EPSP integral. $\boldsymbol{F}$, Peak amplitude and integral of the EPSP in soma (black) and dendrite (red) in D-AP5 expressed in percentage of control conditions for the example shown in $\boldsymbol{A}-\boldsymbol{E}$. G, In an example of focal stimulation, the increase of somatic EPSP amplitude with stimulation strength shows two clear steps both in control ACSF (gray) and with addition of $50 \mu \mathrm{m}$ D-AP5 (black). $\boldsymbol{H}$, Size of observed steps in EPSP amplitude for control ACSF and for $50 \mu \mathrm{M}$ D-AP5. The gray dots indicate the sample average, which is not significantly different in both conditions (paired $t$ test, $p>0.05, n=24$ steps in 8 cells). $\boldsymbol{I}$, Same as $\boldsymbol{H}$ but for the integral of EPSPs. five synapses were enough to generate somatic EPSPs of $>10 \mathrm{mV}$ in smaller neurons (i.e., somatic input resistance $\sim 300 \mathrm{M} \Omega$ ) with an average depolarization at synaptic sites of $20-30 \mathrm{mV}$ and a maximum of $\sim 50 \mathrm{mV}$ (Fig. $8 D$ ). For larger neurons (i.e., somatic 

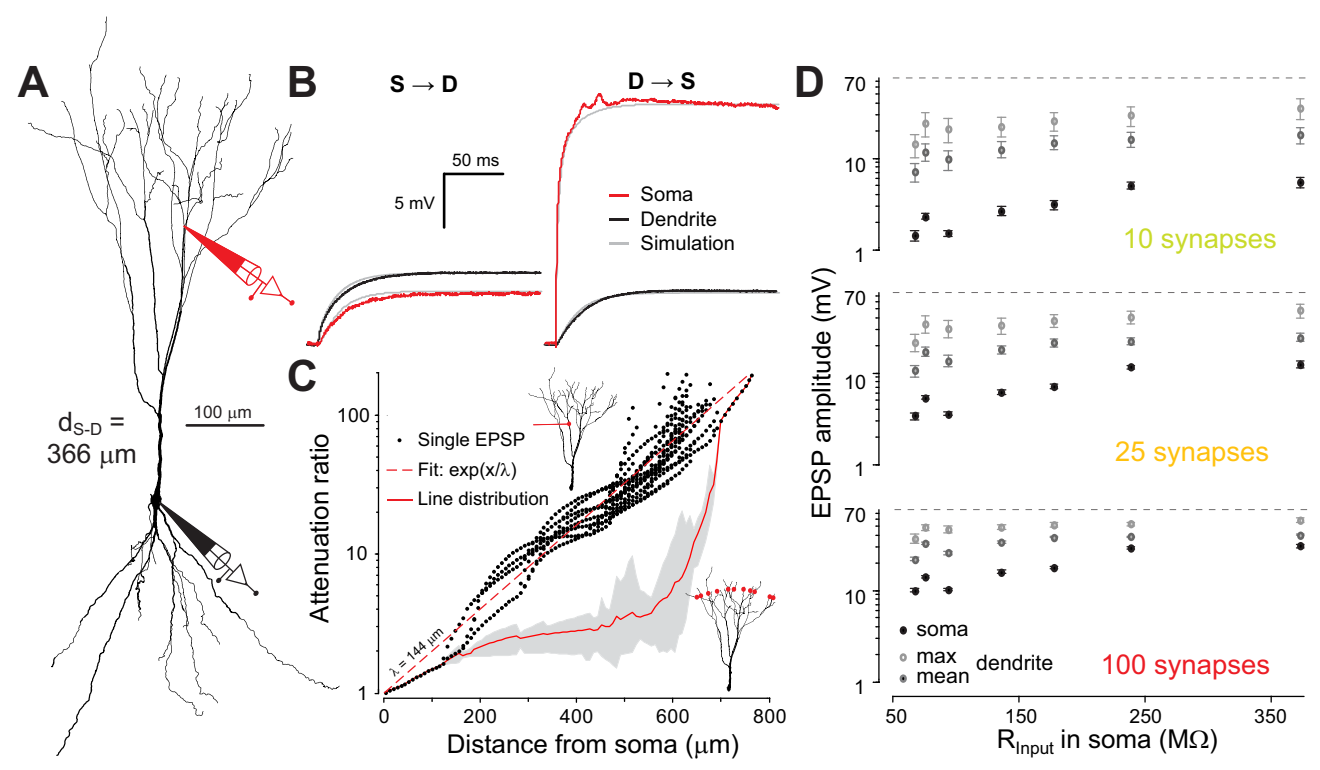

Figure 8. Simulation of passive synaptic integration in pyramidal cells. $A$, Reconstruction of a layer $2 / 3$ pyramidal neuron from which we made simultaneous somatic and dendritic recordings, used here for the simulation. $\boldsymbol{B}$, Voltage response to step current injections at the soma (left) and in the dendrite (right). Simulated traces obtained from best fit of the three passive parameters are superimposed (gray line). C, Black dots, Each dot represents the ratio of dendritic to somatic amplitude (attenuation) for a single EPSC waveform injected in a given dendritic segment. All simulated segments of the apical tree for the neuron shown in $\boldsymbol{A}$ were tested to draw the figure. Red dashed line, Exponential fit of single EPSP attenuation. Gray area and solid red curve, Compound EPSPs arising from contacts located at all dendritic positions equidistant (anatomical path distance) from the soma (see sketch in the bottom right corner where synapses are indicated by red dots). The red curve represents the ratio of the mean dendritic EPSP amplitude across all injection sites to the somatic EPSP amplitude. The shading shows minimum and maximum values observed across each of the locations. D, Voltage at soma (black dots) and at synaptic locations (average over all locations, dark gray; maximum, light gray) for simultaneous activation of 10, 25, or 100 synapses randomly distributed over the apical tree. Results for the seven simulated neurons are displayed as a function of somatic input resistance. Error bars indicate the SD observed over 250 different synaptic distributions.

input resistance $\sim 75 \mathrm{M} \Omega$ ), the same values were reached with 100 synapses (Fig. 8D). Considering that spike threshold is on average $\sim 30 \mathrm{mV}$ above resting potential $(\sim 75 \mathrm{mV})$, we computed that $\sim 75-100$ synapses are necessary to drive smaller neurons to threshold, while $\sim 300-400$ synapses are necessary for larger neurons. This represents between 10 and $20 \%$ of the synapses present on the apical dendrites of layer Ia for large layer III neurons and only $5 \%$ to $10 \%$ of the synapses for semilunar cells, based on a spine density ranging from 40 to 60 units per $100 \mu \mathrm{m}$ (Poo and Isaacson, 2007) and a total dendritic length between 3300 and $5600 \mu \mathrm{m}$ (measured on our reconstructed cells).

On the basis of our experimental findings and the modeling date, we conclude that the apical dendritic tree of piriform cortex neurons can efficiently drive the soma with passive propagation of spatially distributed synaptic inputs.

\section{Spatial distribution of synaptic contacts from single-fiber inputs}

It has been observed that single mitral cell axons sometimes make very strong connections with piriform cortex principal neurons (Franks and Isaacson, 2006). The lack of dendritic electrogenesis in these neurons makes it highly unlikely that such large somatically recorded EPSPs are the result of local dendritic spiking mechanisms. Furthermore, minimal focal stimulation of the dendrites (Fig. 7) suggested that unitary inputs to these dendrites are far smaller than the large EPSPs observed by Franks and Isaacson (2006) at the soma. We are therefore forced to conclude that these steps are the result of single axons projecting many contacts to the same neuron. The question is therefore the extent to which the multiple inputs arising from single axons are clustered or distributed along the dendrites of piriform pyramidal neurons. Passive cable theory predicts that transmission to the soma is more efficient with distributed inputs (Rall, 1970). To test this hypothesis, we used the same minimal LOT stimulation as Franks and Isaacson (2006) and observed similarly sharp increases in EPSP amplitudes in the dendrite and soma $(n=11$ of 24 LOT stimulations paired with dendritic recording) indicating recruitment of individual LOT fibers (e.g., Fig. 9A,B) (Stevens and Wang, 1995). These steps were variable in amplitude and could be as large as $15.4 \mathrm{mV}$ at the soma. In some cases, we could observe $>1$ step in a recording (e.g., Fig. $9 B, C$ ) (note that in these cases the effective attenuation ratio was different for each step, as expected for distinct synaptic inputs). In total, we observed 19 steps during dual recordings. A further constraint on the recordings was that in each case we randomly recorded from one particular dendritic branch. Nonetheless, a clear prediction in the case that inputs were clustered on particular dendritic branches from which we rarely recorded would be that inputs would mostly appear slightly smaller at the dendritic than at the somatic electrode (since attenuation from the soma back into the dendrites is small). We could easily demonstrate this prediction with simulations using NEURON software on cells and conditions reconstructed from our experiments (Fig. 9D). In simulations where we supposed that inputs arose from a single dendritic location, attenuation ratios smaller than 1 were clearly overrepresented (Fig. 9D). In contrast, for the 19 single fibers recorded simultaneously at the soma and at a dendrite, effective attenuation ratios ranged from 0.5 to 4.1 with almost $60 \%$ of them ranging between 1 and 2. This implies that most of the time, some synaptic input contacted the dendrite from which we had recorded which confirms that LOT input typically arises from multiple synaptic contacts distributed widely over the dendrites of piriform cortex pyramidal neurons.

To further test this hypothesis, we compared the trial-to-trial variability of dendritic and somatic EPSP amplitudes. If single fibers contacted the dendritic tree at a single locus, then the ratio 

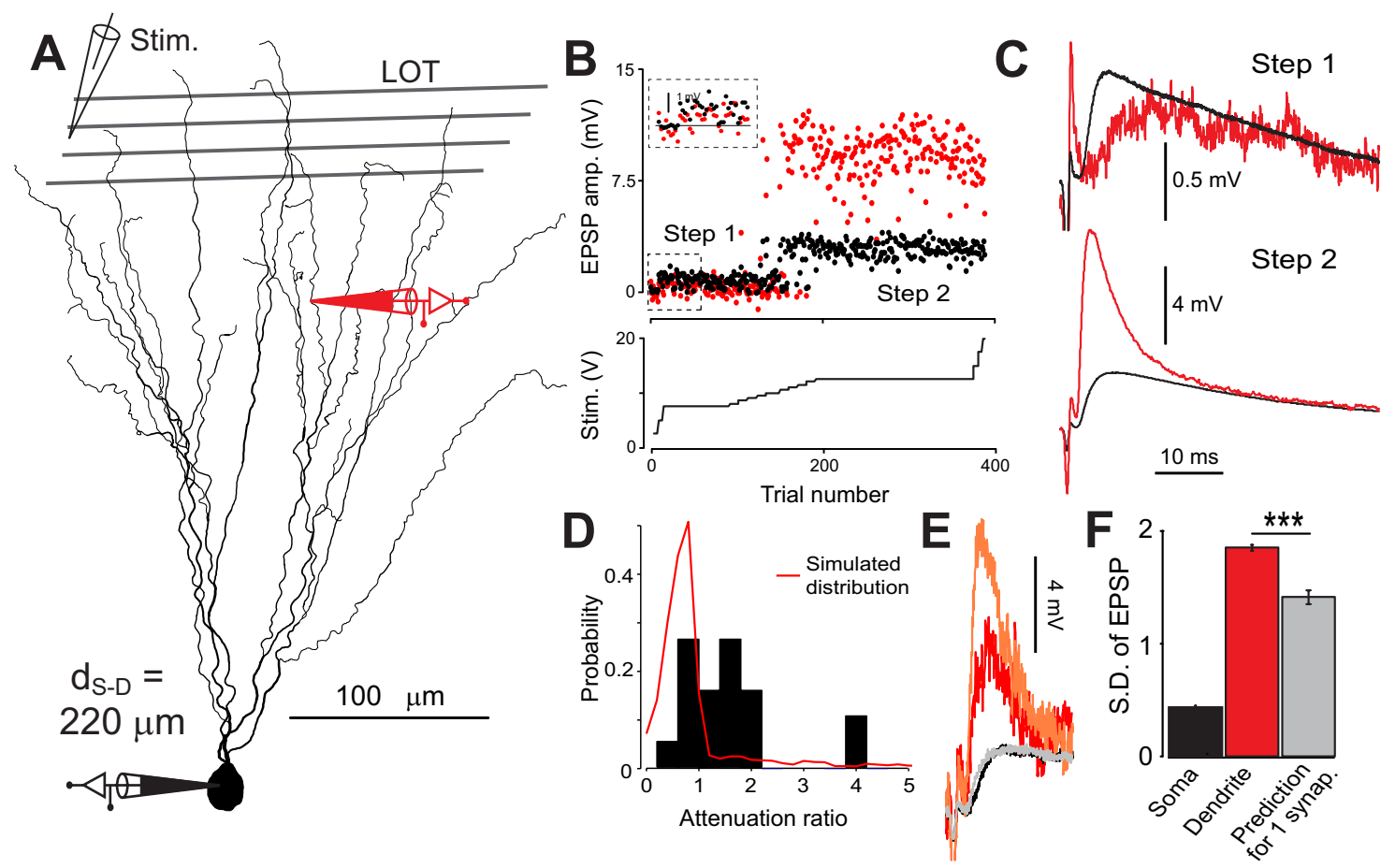

Figure 9. Dendritic response to minimal stimulation. $A$, Position of the recording electrodes (dendritic recording, $d_{S-D}=220 \mu \mathrm{m}$ from soma) on the reconstructed neuron (semilunar cell). A stimulation electrode was placed in the LOT. B, EPSP amplitude in soma (black) and dendrite (red). The stimulus strength was progressively increased (bottom), revealing stepwise changes in EPSP amplitude that corresponded to the recruitment of a new single fiber. The inset shows a magnification of the first amplitude step. C, Mean EPSP waveforms for the two amplitude steps. $\boldsymbol{D}$, Histogram of the distribution of attenuation ratios for the 19 single-fiber responses observed in paired recordings. The red curve shows the expected distribution of attenuation ratio supposing that any single-fiber contacts the dendritic tree at a single locus. All recording distances $(90-435 \mu \mathrm{m}$ ) are pooled together. The two distributions are significantly different (Kolmogorov-Smirnov test, $p=$ $\left.1.3 \times 10^{-6}\right)$. E, Two single-trial responses in the second step displaying the same somatic EPSP amplitude but different dendritic EPSP amplitudes. F, SD of EPSP amplitude at the soma and in the dendrite for the second isolated fiber computed as (variance of second step - variance of first step) ${ }^{0.5}$. The gray bar represents dendritic SD predicted from somatic SD with the assumption that the synaptic input is clustered at a single point of the dendrite. Stars indicate statistically significant difference based on a custom test (see Materials and Methods).

of the SDs of dendritic and somatic EPSP amplitudes is expected to be approximately equal to the ratio of the average dendritic and somatic EPSP amplitudes. If single fibers' synaptic contacts were distributed, we expect the ratio of the SDs to be larger than the ratio of the means (see the statistical analysis of single-fiber experiments in Materials and Methods). We found only one case (Fig. $9 B, C$ ), where the variability at the dendritic recording site was significantly higher than predicted for a single contact (Fig. $9 E, F$ ) (Kolmogorov-Smirnov test, $p=1.4 \times 10^{-12}$, see Materials and Methods). It should be noted that variability analysis is expected to have a low sensitivity in detecting distributed inputs because it requires the dendritic electrode to be positioned such that large differences exists between the effective attenuation ratios associated with each synaptic contacts. This can happen only if a sufficient fraction of the contacts are electrotonically close to the dendritic pipette (distant contacts, e.g., from different primary branches, all give attenuation ratio close to 1 ). Such cases were only rarely met in our experiments: 2 of 19 single fibers gave attenuation ratio larger than 2 . Moreover, the number of repetitions of singlefiber stimulation was limited by the typical duration of the dendritic recording, thereby constraining the sensitivity of the statistics.

Overall, our analysis is in line with the hypothesis that a significant fraction of single fibers make contacts distributed at different loci of the dendritic tree and that LOT input is arranged for the most efficient mode of transmission of coactive inputs.

\section{Discussion}

\section{Active dendritic properties}

We found that apical dendrites of pyramidal cells and semilunar cells of the anterior piriform cortex actively support backpropa- gating APs, local calcium entry, and initiation of local dendritic spikes. Unlike other mammalian pyramidal neurons, we found that spike initiation in the distal dendrites (layer Ia) is unlikely to have any significant effect on the neuron's output. Dendritic spikes in more proximal compartments $(<200 \mu \mathrm{m}$, layer $\mathrm{Ib})$ could nevertheless contribute to somatic spike initiation (Fig. $6 \mathrm{~A}$ ). We found no evidence for local $\mathrm{Ca}^{2+}$ spike initiation as is observed in mammalian neocortex. Though it remains possible that other stimulation methods might lead to NMDA spike generation (e.g., uncaging or iontophoresis of glutamate), we also found no evidence for local NMDA spikes using a stimulation technique known to evoke NMDA spikes in the apical and basal dendrites of neocortical pyramidal cells (Nevian et al., 2007; Larkum et al., 2009). This might be due to the lower density of spines on distal apical dendrites (Poo and Isaacson, 2007) and/or lower density of NMDA channels at LOT synapses (Franks and Isaacson, 2006).

Local spike initiation (e.g., $\mathrm{Na}^{+}, \mathrm{Ca}^{2+}$, and NMDA spikes) has been shown to dominate integrative processes in other mammalian cortical pyramidal neurons (London and Häusser, 2005). It suggests that there is a functional segregation of synaptic integration within the dendritic tree of most pyramidal neurons that highlights the potential importance of synaptic clustering (i.e., convergence of functionally similar input onto dendritic sub-branches; see Larkum and Nevian, 2008). For the principal neurons of the piriform cortex, however, it appears that regenerative events in active subcellular compartments do not contribute to the processing of olfactory bulb input and seems that these cells do not receive clustered, functionally similar LOT input.

All pyramidal neurons thus far studied have been shown to support actively backpropagating APs (Stuart and Sakmann, 
1994; Spruston et al., 1995; Waters et al., 2003) which are accompanied by influx of $\mathrm{Ca}^{2+}$ through voltage-sensitive $\mathrm{Ca}^{2+}$ channels (Markram et al., 1995). Although we find that this is also the case in the piriform cortex cells, the AP amplitudes in trains of backpropagating APs have a highly variable influence on distal dendritic membrane potential which is more pronounced the further the AP travels into the dendritic tree. These in vitro data suggest that distal olfactory bulb synapses may receive an unreliable signal of output activity and spike timing and is consistent with the view that plasticity at LOT synapses is not reliably observed at this age (Poo and Isaacson, 2007).

The variability of backpropagation is an unusual property that has also recently been described in pyramidal neurons of the three-layered turtle pallium (Larkum et al., 2008). Pyramidal neuron dendrites in the turtle pallium, as in the rat piriform cortex, also exhibit predominantly passive properties (Larkum et al., 2008). Since turtles diverged from the species that lead to mammals $\sim 300,000,000$ years ago (Northcutt, 1981), the apparent conservation of dendritic properties between pallium and piriform cortex suggests that implementation of olfaction in mammals did not require specific changes in the computational architecture of principal cortical neurons. In contrast, the more complex active signaling mechanisms in the dendrites of mammalian neocortical pyramidal cells might reflect an evolutionary specialization necessary for higher order function.

\section{Consequences for olfactory coding}

Olfactory information is thought to be encoded in the spatiotemporal patterns of mitral cells (Schaefer and Margrie, 2007; Bathellier et al., 2008) whose axons project diffusely to anterior piriform cortex (Illig and Haberly, 2003) where they contact the distal apical dendrites of pyramidal and semilunar cells. To recognize these patterns the anterior piriform cortex should be able to detect coactivation of select subsets of mitral cells. We did not find any evidence that the apical dendrites of individual pyramidal neurons could efficiently detect and specifically signal coactivated input. This suggests that unique mitral cell activity patterns are not recognized at the subcellular level. However, this feature may arise from connectivity rules between the olfactory bulb and piriform cortex neurons.

Computational studies have suggested that neurons which exhibit dendritic spike mechanisms can be compared with a twolayer neural network (Poirazi et al., 2003). This increased complexity embedded in the cortical network itself can quantitatively improve computational performances such as memory storage capacity (Poirazi and Mel, 2001) or pattern discrimination (Rhodes, 2008). In contrast, our results suggest that a neural network with cells having a single effective threshold provides a computational framework that is sufficient for olfactory processing. One could also speculate that olfactory computations require the detection of large numbers of coactivated mitral cells that necessitate processing information from a number of inputs that exceeds the limits of a single dendritic branch.

The compactness of these dendrites with respect to current loss means that the potency of individual synaptic inputs is relatively location independent (Nevian et al., 2007). It also suggests that spike initiation in the axon is more efficiently driven by synaptic inputs widely distributed over the apical tree. In this scheme and supposing that a spine receives only a single synaptic quanta $(\sim 1 \mathrm{nS})$, we estimate that between 10 and 20\% (300-400 contacts depending on cell size) of the total number of apical dendritic spines in a pyramidal cell would be needed to reach threshold from a resting potential of $-75 \mathrm{mV}$ (see Poo and Isaac- son, 2007, for an evaluation of spine densities). This means that 30-40 LOT fibers would be required to drive piriform pyramidal neurons to fire. This number was smaller in semilunar cells due to their larger input resistance but they still need $\sim 10$ fibers on average to reach threshold (from $-75 \mathrm{mV}$ ).

In conclusion, the dendritic properties of piriform cortex pyramidal neurons lack mechanisms for robust local spike initiation making it unlikely that these types of events play a substantial role in detecting components of complex odor stimuli. We suggest that within the dendritic tree of an individual pyramidal cell, olfactory inputs are randomly located. This arrangement is expected to maximize signaling efficiency (Rall, 1970). The cell-to-cell specificity of such signaling will likely arise from heterogeneity in the combinations of connections between mitral cells and the piriform cortex.

\section{References}

Arbib MA, Erdi P, Szentagothai J (1998) Neural organization structure, function, and dynamics. Cambridge, MA: MIT.

Bathellier B, Buhl DL, Accolla R, Carleton A (2008) Dynamic ensemble odor coding in the mammalian olfactory bulb: sensory information at different timescales. Neuron 57:586-598.

Bi GQ, Poo MM (1998) Synaptic modifications in cultured hippocampal neurons: dependence on spike timing, synaptic strength, and postsynaptic cell type. J Neurosci 18:10464-10472.

Brody CD, Hopfield JJ (2003) Simple networks for spike-timing-based computation, with application to olfactory processing. Neuron 37:843-852.

Carnevale NT, Johnston D (1982) Electrophysiological characterization of remote chemical synapses. J Neurophysiol 47:606-621.

Franks KM, Isaacson JS (2006) Strong single-fiber sensory inputs to olfactory cortex: implications for olfactory coding. Neuron 49:357-363.

Froemke RC, Poo MM, Dan Y (2005) Spike-timing-dependent synaptic plasticity depends on dendritic location. Nature 434:221-225.

Harris NC, Constanti A (1995) Mechanism of block by ZD 7288 of the hyperpolarization-activated inward rectifying current in guinea pig substantia nigra neurons in vitro. J Neurophysiol 74:2366-2378.

Hasselmo ME, Wilson MA, Anderson BP, Bower JM (1990) Associative memory function in piriform (olfactory) cortex: computational modeling and neuropharmacology. Cold Spring Harb Symp Quant Biol 55:599-610.

Häusser M, Major G, Stuart GJ (2001) Differential shunting of EPSPs by action potentials. Science 291:138-141.

Illig KR, Haberly LB (2003) Odor-evoked activity is spatially distributed in piriform cortex. J Comp Neurol 457:361-373.

Kampa BM, Letzkus JJ, Stuart GJ (2007) Dendritic mechanisms controlling spike-timing-dependent synaptic plasticity. Trends Neurosci 30:456463.

Larkum ME, Nevian T (2008) Synaptic clustering by dendritic signalling mechanisms. Curr Opin Neurobiol 18:321-331.

Larkum ME, Watanabe S, Lasser-Ross N, Rhodes P, Ross WN (2008) Dendritic properties of turtle pyramidal neurons. J Neurophysiol 99:683-694.

Larkum ME, Nevian T, Sandler M, Polsky A, Schiller J (2009) Synaptic integration in tuft dendrites of layer 5 pyramidal neurons: a new unifying principle. Science 325:756-760.

Lin DY, Zhang SZ, Block E, Katz LC (2005) Encoding social signals in the mouse main olfactory bulb. Nature 434:470-477.

London M, Häusser M (2005) Dendritic computation. Annu Rev Neurosci 28:503-532.

Magee JC, Johnston D (1997) A synaptically controlled, associative signal for Hebbian plasticity in hippocampal neurons. Science 275:209-213.

Margrie TW, Schaefer AT (2003) Theta oscillation coupled spike latencies yield computational vigour in a mammalian sensory system. J Physiol 546:363-374.

Margulis M, Tang CM (1998) Temporal integration can readily switch between sublinear and supralinear summation. J Neurophysiol 79:2809-2813.

Markram H, Helm PJ, Sakmann B (1995) Dendritic calcium transients evoked by single back-propagating action potentials in rat neocortical pyramidal neurons. J Physiol 485:1-20. 
Markram H, Lübke J, Frotscher M, Sakmann B (1997) Regulation of synaptic efficacy by coincidence of postsynaptic APs and EPSPs. Science 275:213-215.

Mel BW (1993) Synaptic integration in an excitable dendritic tree. J Neurophysiol 70:1086-1101.

Migliore M, Shepherd GM (2002) Emerging rules for the distributions of active dendritic conductances. Nat Rev Neurosci 3:362-370.

Mori K, Takahashi YK, Igarashi KM, Yamaguchi M (2006) Maps of odorant molecular features in the mammalian olfactory bulb. Physiol Rev 86:409-433.

Nevian T, Larkum ME, Polsky A, Schiller J (2007) Properties of basal dendrites of layer 5 pyramidal neurons: a direct patch-clamp recording study. Nat Neurosci 10:206-214.

Neville KR, Haberly LB (2004) Olfactory cortex. In: The synaptic organization of the brain. Oxford: Oxford UP.

Northcutt RG (1981) Evolution of the telencephalon in nonmammals. Annu Rev Neurosci 4:301-350.

Poirazi P, Mel BW (2001) Impact of active dendrites and structural plasticity on the memory capacity of neural tissue. Neuron 29:779-796.

Poirazi P, Brannon T, Mel BW (2003) Pyramidal neuron as two-layer neural network. Neuron 37:989-999.

Polsky A, Mel BW, Schiller J (2004) Computational subunits in thin dendrites of pyramidal cells. Nat Neurosci 7:621-627.

Poo C, Isaacson JS (2007) An early critical period for long-term plasticity and structural modification of sensory synapses in olfactory cortex. J Neurosci 27:7553-7558.

Rall W (1970) Cable properties of dendrites and effects of synaptic location. In: Excitatory synaptic mechanisms (Anderson P, Jansen JKS, eds), pp 175-186. Oslo: Universetetesforlag.

Rall W, Rinzel J (1973) Branch input resistance and steady attenuation for input to one branch of a dendritic neuron model. Biophys J 13:648-687.

Rhodes PA (2008) Recoding patterns of sensory input: higher-order fea- tures and the function of nonlinear dendritic trees. Neural Comput 20:2000-2036.

Rinzel J, Rall W (1974) Transient response in a dendritic neuron model for current injected at one branch. Biophys J 14:759-790.

Schaefer AT, Margrie TW (2007) Spatiotemporal representations in the olfactory system. Trends Neurosci 30:92-100.

Schiller J, Major G, Koester HJ, Schiller Y (2000) NMDA spikes in basal dendrites of cortical pyramidal neurons. Nature 404:285-289.

Sciancalepore M, Constanti A (1998) Inward-rectifying membrane currents activated by hyperpolarization in immature rat olfactory cortex neurones in vitro. Brain Res 814:133-142.

Sjöström PJ, Häusser M (2006) A cooperative switch determines the sign of synaptic plasticity in distal dendrites of neocortical pyramidal neurons. Neuron 51:227-238.

Spruston N, Schiller Y, Stuart G, Sakmann B (1995) Activity-dependent action-potential invasion and calcium influx into hippocampal CA1 dendrites. Science 268:297-300.

Stevens CF, Wang Y (1995) Facilitation and depression at single central synapses. Neuron 14:795-802.

Stuart GJ, Sakmann B (1994) Active propagation of somatic actionpotentials into neocortical pyramidal cell dendrites. Nature 367:69-72.

Stuart G, Schiller J, Sakmann B (1997) Action potential initiation and propagation in rat neocortical pyramidal neurons. J Physiol 505:617-632.

Suzuki N, Bekkers JM (2006) Neural coding by two classes of principal cells in the mouse piriform cortex. J Neurosci 26:11938-11947.

Waters J, Helmchen F (2004) Boosting of action potential backpropagation by neocortical network activity in vivo. J Neurosci 24:11127-11136.

Waters J, Larkum M, Sakmann B, Helmchen F (2003) Supralinear $\mathrm{Ca}^{2+}$ influx into dendritic tufts of layer $2 / 3$ neocortical pyramidal neurons in vitro and in vivo. J Neurosci 23:8558-8567.

Zhou WL, Yan P, Wuskell JP, Loew LM, Antic SD (2008) Dynamics of action potential backpropagation in basal dendrites of prefrontal cortica pyramidal neurons. Eur J Neurosci 27:923-936. 